ARCHIVO ESPAÑOL DE ARTE, LXXXIX, 353

ENERO-MARZO 2016, pp. 77-84

ISSN: 0004-0428, eISSN: 1988-8511

doi: $10.3989 /$ aearte.2016.06

\title{
VARIA
}

\section{UN NUEVO DIBUJO DE FRANCISCO PACHECO PARA SU LIBRO DE RETRATOS: LA EFIGIE DE PABLO DE CÉSPEDES EN LOS UFFIZI}

\author{
Benito Navarrete Prieto \\ Universidad de Alcalá ${ }^{1}$ \\ benito.navarrete@uah.es
}

\begin{abstract}
Se da a conocer aquí un dibujo autógrafo de Francisco Pacheco, retrato del pintor Pablo de Céspedes y relacionado con el Libro de retratos de aquél. Este dibujo es la fuente original que siguieron las cinco copias conservadas en la Hispanic Society of America de Nueva York, en el manuscrito de la Real Biblioteca de El Escorial, en la colección Anmunátegui, y dos en la Biblioteca Nacional de España, una de mano de Francisco de Goya. El dibujo, atribuido hasta ahora a Barocci en el Gabinete de Dibujos y Estampas de los Uffizi, procede de la prestigiosa donación Santarelli. El hallazgo supone la constatación de la importancia de las copias de la Hispanic Society, que abren la posibilidad de que existan otros originales de Pacheco aún no identificados.
\end{abstract}

Palabras clave: Francisco Pacheco; Barocci; Libro de Retratos; Uffizi; Santarelli; Dibujo español.

\section{A NEW DRAWING BY FRANCISCO PACHECO FOR HIS LIBRO DE RETRATOS: THE PORTRAIT OF PABLO DE CÉSPEDES IN THE UFFIZI}

The author presents an original drawing by Francisco Pacheco, a portrait of the painter Pablo de Céspedes, related to Pacheco's famous Libro de retratos. The drawing is the original of five copies by different hands kept at The Hispanic Society of America (Nueva York), the Royal Library of the Escorial, and the Amunátegui Collection; two copies are in the National Library of Spain, one of which is by Francisco de Goya.

The drawing under consideration was formerly attributed to Barocci and comes from the prestigious collection donated by Santarelli to the Uffizi Gallery in the 19th century. This discovery highlights the importance of the copies conserved at The Hispanic Society and suggests there may be more unidentified portraits by Pacheco.

Key words: Francisco Pacheco; Barocci; Libro de retratos; Uffizi; Santarelli; Spanish drawing.

\footnotetext{
${ }^{1}$ Este artículo se inscribe dentro del proyecto de investigación promovido por la Fundación Mapfre gracias al convenio firmado con el Gabinete de dibujos y estampas de los Uffizi. Agradezco a Pablo Jiménez Burillo, Mónica Fuentes, Nadia Arroyo y María López la confianza depositada, así como a Marzia Faietti y Giorgio Marini las facilidades otorgadas. Durante el proceso de estudio ha sido fundamental el trabajo de investigación en el Kunsthistorisches Institut de Florencia como Ospite scientifico, gracias a la acogida de sus directores Alessandro Nova y Gerhard Wolf, a quienes también extiendo mi agradecimiento, así como a Manuela Mena, Juan Montero y José Solis. El autor es miembro del Grupo de Investigación HAR2014-52061-P La copia pictórica en la Monarquía Hispánica (Siglos XVI-XVIII) del programa estatal de fomento de la investigación científica y técnica de excelencia. Subprograma Estatal de Generación del Conocimiento del Ministerio de Economía y Competitividad del que es investigador principal David García Cueto.
} 
En 1979 la Hispanic Society de Nueva York adquirió en subasta pública ${ }^{2}$ un interesante conjunto de diez dibujos de calidad desigual que fueron relacionados por diferentes autores como directamente derivados o vinculables con el Libro de descripción de verdaderos retratos de ilustres y memorables varones de Francisco Pacheco. Cuando fueron publicados en el Corpus of Spanish drawings ${ }^{3}$, ocho de estos dibujos se consideraron como originales de Pacheco, concretamente los correspondientes a: César Arbasia, Luis Fernández, Juan Fernández Navarrete "el Mudo", Felipe de Liaño, Diego Velázquez, y ante la duda se propusieron como "atribuidos a" los identificados como Pablo de Céspedes y Jerónimo Hernández. De este grupo fueron excluidos como autógrafos de Pacheco tanto la efigie de Alonso Cano como la de Antonio del Castillo que correspondían evidentemente a una cronología más avanzada. Sería sin embargo la hispanista Priscilla E. Muller la primera que pondría en duda esta aseveración, al considerar que, al menos el de Luis de Vargas, era claramente una copia del siglo XVII del libro de retratos ${ }^{4}$. Posteriormente Pérez Sánchez los consideró igualmente como copias ${ }^{5}$, y Bonaventura Bassegoda, que en un primer momento los puso en relación con Pacheco y con un hipotético libro monográfico de Elogios de $\operatorname{artistas}^{6}$, finalmente se retractó de su idea, considerándolos claramente como copias del siglo XVIII ${ }^{7}$. Apuntaba entonces la sugestiva idea de que los dibujos de la Hispanic Society estuvieran en relación con los trabajos de ampliación e ilustración de la segunda edición de las Vidas de Palomino, que finalmente se reimprimieron sin ilustraciones en 1795-1797. En otro trabajo repleto de erudición Bassegoda ${ }^{8}$ insistía en esta idea, concluyendo una hipótesis bien interesante, y es que estas copias de artistas estarían promovidas probablemente por Antonio Ponz o Isidoro Bosarte, intentando crear una galería de artistas españoles para completar así la primera edición del Palomino en su segunda edición.

Por su parte Priscilla E. Muller ha vinculado estas copias, procedentes de la colección de Stirling-Maxwell (1818-1878), con su proyecto de ilustrar los Annals of the Artists of Spain que se publicó en Londres en 1847-1848. No tendría nada de extraño que el propio Stirling los hubiera adquirido de Ceán Bermúdez y que previamente estos dibujos hubiesen servido como material de documentación iconográfica para Goya en un más que posible encargo para ilustrar el Diccionario de Ceán, y en relación al cual Pérez Sánchez publicó en $1993^{9}$ otros dos dibujos del pintor aragonés: el de Jerónimo Hernández y el de Pablo de Céspedes que derivan igualmente de los de la Hispanic. El tema es apasionante pues de facto este conjunto de dibujos se antoja como uno de los documentos de primer orden para demostrar que las copias de Pacheco o los originales del artista descartados circularon bastante. Marta Cacho ${ }^{10}$ señaló que el Libro de Pacheco circuló como fuente iconográfica durante el siglo XVII y XVIII, aspecto sobre el que no todos los autores están de acuerdo ${ }^{11}$. Se podría elucubrar que los dibujos de la Hispanic Society derivarían directamente de los originales

Christie's sale catalogue of Old Master Drawings, 28 de marzo de 1979, lotes 242-248.

Angulo/ Pérez Sánchez, 1985: cat. 197-204: 51-53.

4 Angulo/ Pérez Sánchez, 1985: cat n 242: 47. Un análisis completo sobre toda la serie y un meticuloso estudio técnico sobre las filigranas de primer soporte y de segundo soporte de los mismos fue hecho por Muller, 2006: 69-101.

${ }^{5}$ Pérez Sánchez, 1993: 167-176.

${ }^{6}$ Bassegoda i Hugas, 1991: 188-189.

${ }^{7}$ Bassegoda i Hugas, Bonaventura, 2000-2001: 205-206. Consideramos este artículo una aportación fundamental para valorar la importancia del Libro de Pacheco y el estatus e importancia del ejemplar conservado en el museo Lázaro Galdiano, así como por la identificación de dos de los retratados: Francisco de Rioja y Diego Hurtado de Mendoza; además suministra la fuente para la futura identificación del retrato del tío y protector del pintor, el canónigo Francisco Pacheco.

${ }^{8}$ Bassegoda i Hugas, 2003: 108-109.

9 Pérez Sánchez, 1993. También Bassegoda ha relacionado estas copias de Goya con el proyecto del Diccionario de Ceán. Véase Bassegoda, 2003: 108.

${ }^{10}$ Cacho Casal, 2011: 214.

${ }^{11}$ Bassegoda i Hugas, 2012: 179 y 2015: 38-39.

Arch. esp. arte, LXXXIX, 353, ENERO-MARZO 2016, 77-84 ISSN: 0004-0428, eISSN: 1988-8511, doi: 10.3989/aearte.2016.06 
de Pacheco que se perdieron, y son precisamente estos dibujos desaparecidos los que presumiblemente formarían parte del Libro, o se relacionaron directamente como material preparatorio o borradores y por tanto sería el material que estaría al alcance de los eruditos, aficionados o coleccionistas $^{12}$. De ese modo, podemos suponer también que Pacheco realizaría las efigies de César Arbasia, Juan Fernández Navarrete "el Mudo", Jerónimo Hernández e incluso Diego Velázquez, a algunos de los cuales sabemos por el Arte de la Pintura que les compuso también elogios.

Muller llegó también a plantear con cautela que el correspondiente a Pablo de Céspedes pudiera ser autógrafo de Pacheco, aunque se inclinaba más en que pudiera ser de un seguidor del artista del segundo tercio del siglo XVII ${ }^{13}$. La semejanza técnica del soporte y la grafía de las inscripciones con respecto al retrato de Arbasia, le permitió incluso sugerir que ambos podrían corresponder a la misma mano, que vinculaba con el cordobés Juan de Alfaro y Gámez, pues por Palomino sabemos que hizo retratos de medio cuerpo de hombres ilustres por encargo de Pedro de Arce (1607-1656). Lo cierto es que la identidad del efigiado en este dibujo también ha sido puesta en duda, a pesar de tener una inscripción antigua, probablemente del siglo XVII, en la que se puede leer "Pablo de Cespedes. Racionero de la Sta Iglesia/de Cordoba Pintor Famoso". Las dudas en la identificación estaban fundamentadas en la representación que eligió finalmente Pacheco para incluir a su amigo Céspedes en su Libro y que no se corresponde con la que aparece en el dibujo de la Hispanic, aunque a nuestro juicio y al de otros autores, sí podría representar a la misma persona pero más madura. Hasta el momento presente, el dibujo del museo neoyorquino era creído como el original del que habían derivado hasta cuatro copias, todas ellas directamente relacionadas con la intención de ilustrar con retratos auténticos algunas de las ediciones con repertorios de artistas españoles, de sus vidas o del diccionario de Ceán.

El trabajo de investigación previo a la catalogación de los dibujos españoles de los Uffizi y la revisión que estamos haciendo de todos los fondos del gabinete de dibujos y estampas (GDSU), incluidos los considerados italianos, nos ha permitido localizar entre los retratos atribuidos a Federico Barocci ${ }^{14}$ uno de hombre sin identificar (fig. 1), procedente del importante fondo donado en 1866 por Emilio Santarelli ${ }^{15}$ (1801-1886) y que, sin lugar a dudas, es el original de Francisco Pacheco del cual deriva el de la Hispanic Society ${ }^{16}$ (fig. 2) y probablemente después el del manuscrito de la Biblioteca Real de San Lorenzo de El Escorial. Como en los otros retratos del libro conservado en el Museo Lázaro Galdiano, se trata de un dibujo realizado a lápiz negro y sanguina sobre papel verjurado, siendo de menores dimensiones $(113 \times 90 \mathrm{~mm})$ al tratarse tan solo del rostro y comienzo del busto y probablemente se haya cortado en fechas que desconocemos. Su fondo presenta la cuadrícula reticular menuda que se advierte en otros dibujos como en el de Francisco de Rioja identificado por Bassegoda, y por el resto de sus características técnicas no puede haber reparo en que estamos ante el original perdido de uno de los dos retratos que hizo Pacheco de su amigo Céspedes.

\footnotetext{
${ }^{12}$ En este ámbito de los borradores o dibujos descartados para su Libro de Retratos es donde habría que ubicar también los conservados en la Real Biblioteca de Madrid y en la Biblioteca Nacional de España. Sobre las vicisitudes e historia material de estos retratos, y acerca de la hipótesis bastante interesante de la procedencia del grupo de la Real Biblioteca de la colección del Conde del Águila, véase: Cacho Casal, 2010: 447-455.

${ }_{13}$ Muller, 2006: cat. $\mathrm{n}^{\circ}$ 16: 80-83.

${ }^{14}$ Gabinete de dibujos y estampas de los Uffizi, no 9328 S. Dibujo a lápiz negro y sanguina sobre papel verjurado, 113 x $90 \mathrm{~mm}$. Con inscripción a lápiz en la parte superior “21". Está inserto dentro de un paspartú con un conjunto de seis dibujos en total entre las carpetas de Barocci. Agradezco a Paola Grifoni, Secretaria Regional de la ex Soprintendenza per il Polo Museale de la ciudad de Florencia, el permiso administrativo para poder reproducir esta obra.

${ }^{15}$ Santarelli, 1870: 637: $n^{\circ} 20$ Fiori Federigo detto il Baroccio "Testa di un frate, vista in faccia. Matita rossa e nera; carta idem”. En su inventario manuscrito consta el precio de adquisición en 8 liras.

${ }^{16}$ Agradezco a Patrick Lenaghan el permiso administrativo de publicación y la fotografía de este dibujo propiedad de la Hispanic Society of America.
}

Arch. esp. arte, LXXXIX, 353, ENERO-MARZO 2016, 77-84

ISSN: 0004-0428, eISSN: 1988-8511, doi: 10.3989/aearte.2016.06 


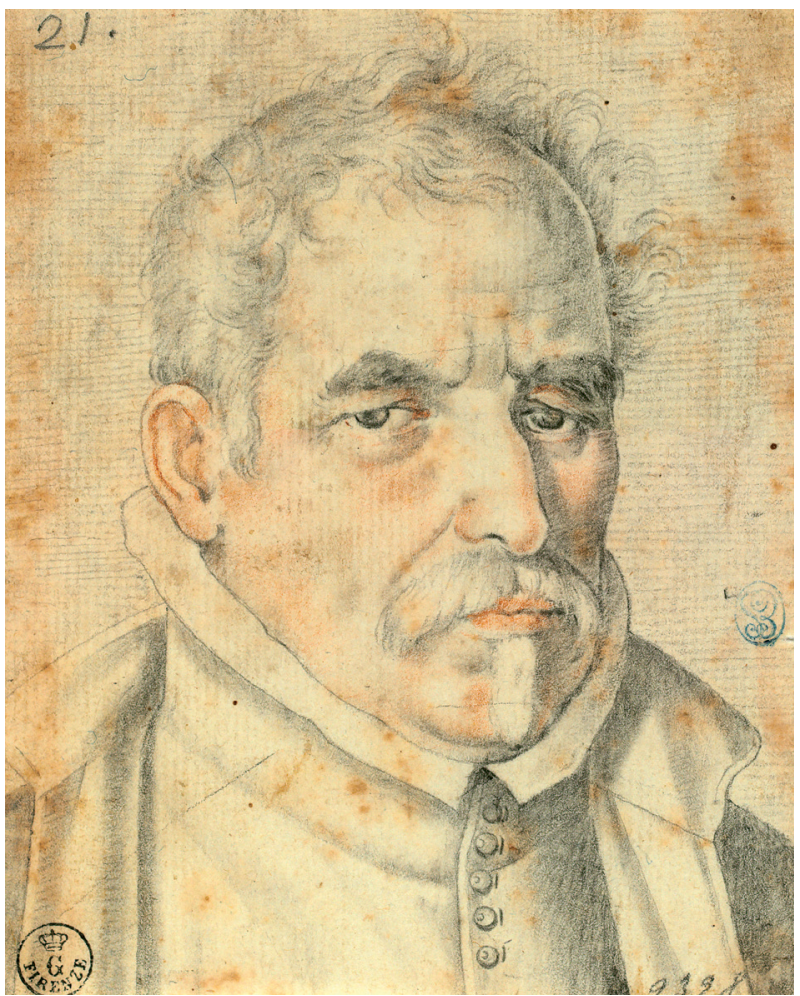

Fig. 1. Francisco Pacheco, Retrato de Pablo de Céspedes, Lápiz negro y sanguina sobre papel verjurado, 113 x $90 \mathrm{~mm}$, Florencia, Gabinetto Disegni e Stampe degli Uffizi, nº 9328S.

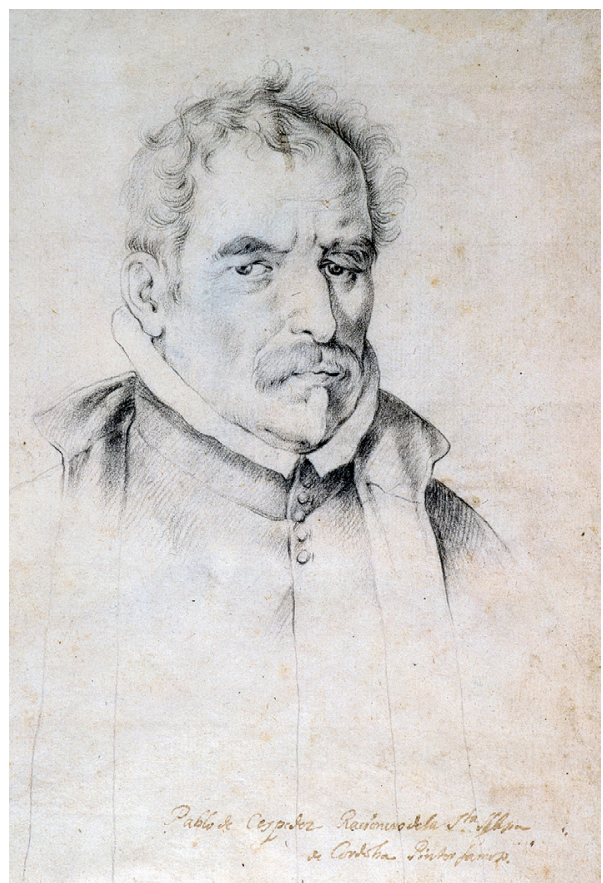

Fig. 2. Anónimo del segundo tercio del siglo XVII, ¿Juan de Alfaro y Gámez?, copia de Francisco Pacheco, Retrato de Pablo de Céspedes, Lápiz negro sobre papel verjurado, 199 x 138 mm, Nueva York, The Hispanic Society of America, Acc. $N^{\circ}$ LA428.

El primer problema que nos asalta al estudiar el dibujo es la identidad del retratado ${ }^{17}$. La antigua inscripción del dibujo de la Hispanic es un poderoso elemento para no dudar de su identificación con Céspedes, al igual que su evidente relación con el retrato que Pacheco sí incluyó en su Libro, solo que en la hoja de los Uffizi lo presenta de mayor edad y con menos cabello, con bigote y perilla, y la mirada girada a la derecha no a la izquierda. Viste pesados ropajes de amplios pliegues, eligiendo Pacheco la misma indumentaria que para el retrato de Rioja, incluso con los cinco botones y la huella de los ojales idénticos, detalle que, al igual que la costura del doblez de su gabán o sobretodo, y de los perfiles del alzacuello, van a ser claves para poder concluir la importancia de este dibujo y definir la gradación y estatus de las copias en el espacio y en el tiempo.

Fue Muller ${ }^{18}$ quien estableció la ordenación de las hasta ahora cinco copias conocidas, cuya secuencia, añadiendo el original ahora dado a conocer, en virtud de la seriación y de los nuevos elementos a considerar, sería:

\footnotetext{
${ }^{17}$ Las primeras dudas sobre la identidad del retratado, hicieron contemplar por indicación de José Solís y Juan Montero, si no pudiera tratarse de la efigie de su tío Francisco Pacheco, que por múltiples razones de agradecimiento debió retratar. Sin embargo el retrato que suministra el manuscrito de El Escorial y que puede verse en el artículo de Bassegoda, 2000-2001: p. 216, fig. 9, nos ha hecho descartar esta hipótesis, lo que no impide que el original de Pacheco de la efigie de su tío pueda aparecer en el futuro. Agradezco a Juan Montero, José Solís y Bonaventura Bassegoda sus apreciaciones en este sentido.

${ }^{18}$ Muller, 1996: 91.
} 
$1^{\circ}$ El dibujo autógrafo y original de Francisco Pacheco conservado en los Uffizi que ahora se da a conocer: 113 x $90 \mathrm{~mm}$.

$2^{\circ}$ El dibujo conservado en la Hispanic Society, copia realizada en la segunda mitad del siglo XVII: 199 x $138 \mathrm{~mm}$.

$3^{\circ}$ El dibujo conservado dentro de un libro de grabados en la Real Biblioteca de El Escorial, probablemente del siglo XVIII: 124 x $90 \mathrm{~mm}^{19}$.

$4^{\circ}$ El dibujo conservado en la Biblioteca Nacional, anteriormente en la colección de Carderera y que consideramos también del siglo XVIII: 198 x $143 \mathrm{~mm}$.

$5^{\circ}$ El de la colección Anmunátegui que ha de considerarse como copia de un anónimo artista del XVIII: 219 x $156 \mathrm{~mm}^{20}$.

6 El de Goya conservado en la Biblioteca Nacional y que también fue propiedad de Carderera: $173 \times 127 \mathrm{~mm}$.

Todas estas copias derivan del dibujo original que hemos encontrado entre los dibujos donados a los Uffizi en 1866 por Santarelli y que, una vez más, encuentran un vínculo con las colecciones de la Biblioteca Nacional de España.

Si comparamos el retrato de Pacheco de los Uffizi con el de la Hispanic, lo primero que advertimos es que ha sabido transferirse la impronta de seguridad y firmeza en el rostro del retratado. Esto se percibe igualmente en el dibujo del platero Ballesteros perteneciente al libro del Lázaro Galdiano, con el que igualmente se relaciona técnicamente el presente dibujo. Vemos unos perfiles perfectos con la impronta de la sanguina en pómulos, labios, mentón y las arrugas en la frente.

Sin embargo, advertimos que la copia de la Hispanic Society únicamente no es fiel en la trasposición de la costura que hace Pacheco en la zona del cuello del sobretodo, en la parte derecha del retrato, donde el trazo lineal se torna quebrado, vacilante. Esa forma accidentada de la costura se va a mantener en las cinco copias posteriores. Pensamos de hecho que muy probablemente el dibujo de la Hispanic podría haber sido realizado en la segunda mitad del siglo XVII, y que tanto éste dibujo como el de Arbasia y los cercanos a este grupo, sirvieron como material que luego conocería muy probablemente Palomino y desde luego Ceán. Así pues, el dibujo de la Biblioteca Nacional que perteneció a Valentín Carderera ${ }^{21}$ y que presenta una cuadricula en el rostro del pintor (fig. 3), podría ser el que se relacionara con los trabajos preparatorios de ilustración del Diccionario de Ceán y, por consiguiente, el que serviría a Goya como documentación iconográfica para el suyo que, a la postre, termina siendo una imagen inspirada en este pero mucho más dramatizada y enfática ${ }^{22}$ (fig. 4).

\footnotetext{
${ }^{19}$ El manuscrito de El Escorial fue catalogado por Gregorio de Andrés como "Dibujos de retratos de hombres ilustres dentro de un libro de grabados". Sig. 28.I.14 de la Real Biblioteca de El Escorial. Indicaba entonces que los dibujos estaban artificialmente pegados en un libro de grabados probablemente en el siglo XIX. Sin ninguna razón aparente los atribuyó con interrogante a Antonio Ponz. El correspondiente a Céspedes -que copia igualmente el dibujo de la Hispanic- reza en la inscripción: Andrés, 1968: folio 17: n 17: "Pablo Cespedez Razionero de la Sa. Iglesia de Córdoba pintor famoso". Las dimensiones del dibujo son de 124 x 90 mm. De todos es el más fiel en su copia al dibujo de la Hispanic. Agradezco al profesor de la Universidad de Sevilla José Solís el envío de la copia del manuscrito para poder cotejar la información.

${ }^{20}$ Así lo estudió Pérez Sánchez, 1993: 173-174, inclinándose por atribuirlo a un anónimo sevillano del siglo XVIII que haría esas copias por encargo de Ceán para proporcionar material al futuro Diccionario.

${ }^{21}$ Santiago, 1966: vol. I: 613, nº 2079.7.

${ }^{22}$ Sobre todo esto es imprescindible el artículo de Pérez Sánchez, 1993 y los previos de Salas, 1962: 411-413; 1964 : 14-19. Como señaló Pérez Sánchez no se pueden aceptar todas las propuestas de Salas en este artículo. Más riguroso es el de Held, 1966: 294-298. La circunstancia de que las copias de los dibujos previos hayan servido como material de documentación para proyectos diferentes fue también señalado por Bassegoda, 2003: 109. Por tanto es Goya con su particular estilo el que les da unidad.
}

Arch. esp. arte, LXXXIX, 353, ENERO-MARZO 2016, 77-84

ISSN: 0004-0428, eISSN: 1988-8511, doi: 10.3989/aearte.2016.06 


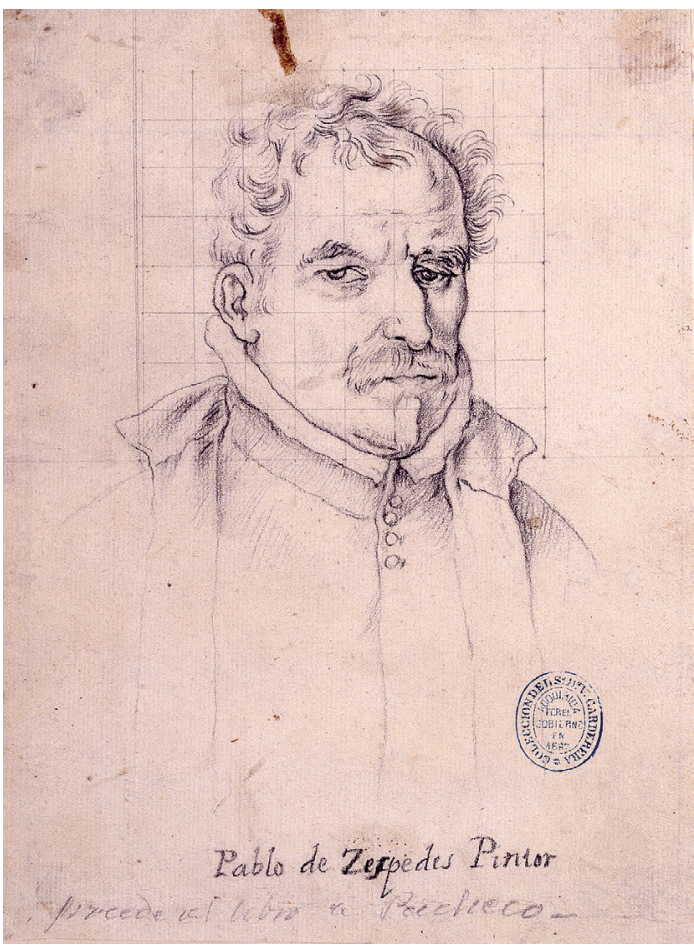

Fig. 3. Anónimo del siglo XVIII, copia de Francisco Pacheco, Retrato de Pablo de Céspedes, Lápiz negro sobre papel verjurado, 198 x $143 \mathrm{~mm}$, Madrid, Biblioteca Nacional de España, Dib/15/29/23.

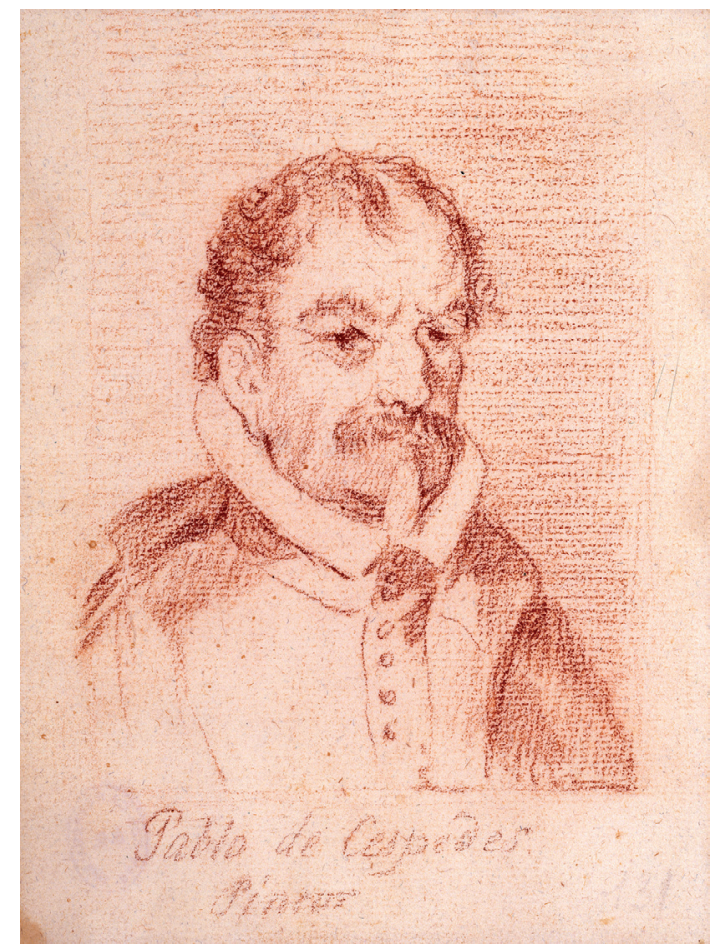

Fig. 4. Francisco de Goya, copia de Francisco Pacheco, Retrato de Pablo de Céspedes, Sanguina sobre papel verjurado, 173 x $127 \mathrm{~mm}$, Madrid, Biblioteca Nacional de España, Dib/18/1/7840.

Más interesante todavía resulta incidir en la inscripción que presenta la copia de la Biblioteca Nacional donde Carderera escribe: "Procede del Libro de Pacheco". Diferentes autores han calificado como erróneo este dato, algo que a todas luces y como ahora pretendemos demostrar, debe ser contemplado, al menos, como probable.

Y la pregunta que nos hacemos es: ¿perteneció este nuevo dibujo de los Uffizi al Libro de retratos de Pacheco tal y como lo concebimos hoy en el Libro conservado en el Museo Lázaro Galdiano? Pensamos que desde luego este dibujo está directamente relacionado con este proyecto y se podría vincular con el que cita en su elogio del Libro diciendo que le hizo al racionero cordobés, "honor de España, Céspedes divino..., en una destas venidas (a Sevilla) siendo mi guesped lo retrate i le hize un soneto..."23.

Otra cuestión interesante y conflictiva que no se nos escapa, es la relevancia que tiene que existan dos retratos diferentes para un mismo personaje y relacionables con el Libro, algo realmente excepcional. Pensamos que en este caso la razón estriba en la relación personal de ambos artistas. No en vano Céspedes fue uno de los principales informantes para Pacheco, de cuyo material se

\footnotetext{
${ }^{23}$ Pacheco, [1599]1983: 64-66.
} 
sirvió no solo en su Libro de retratos sino también en su Arte de la Pintura ${ }^{24}$. El hecho de que haya aparecido este nuevo dibujo puede apuntalar la teoría de Bassegoda acerca de que realmente el Libro que hoy conserva el Lázaro Galdiano es el que Pacheco concibió ${ }^{25}$, y que los dibujos que se dispersaron o desmembraron no eran realmente los que el artista dio por definitivos para su obra sino bocetos, borrones o tanteos, lo que puede explicar la aseveración del propio Pacheco al decir que "bien pasarán de ciento y setenta" ${ }^{26}$. Así es como entiendo que hay que considerar este nuevo dibujo del que no tengo ninguna duda de su carácter autógrafo y cuya importancia concede ahora si cabe más relevancia al estatus de los dibujos de la Hispanic Society que, al menos en los que tienen un mayor aire de familia, se podría pensar que son copias de originales perdidos que, como este de los Uffizi, podrían aparecer en el futuro.

\section{BIBLIOGRAFÍA}

Andrés Martínez, Gregorio de (1968): "Catálogo de las colecciones de dibujos de la Real Biblioteca de El Escorial”. En: Archivo Español de Arte, 161, pp. 3-19.

Angulo Íniguez, Diego / Pérez Sánchez, Alfonso Emilio (1985): A Corpus of Spanish Drawings. Volume Three: Seville 1600-1650. Londres: Harvey Miller Publishers.

Bassegoda i Hugas, Bonaventura (1991): "Cuestiones de iconografía en el Libro de retratos de Francisco Pacheco". En: Cuadernos de Arte e Iconografía, Tomo IV, nº 7, pp. 186-196.

Bassegoda i Hugas, Bonaventura (2000-2001): "El libro de retratos de Pacheco y la verdadera efigie de don Diego Hurtado de Mendoza”. En: Locus Amoenus, n 5, pp. 205-216.

Bassegoda i Hugas, Bonaventura (2003): "Antonio Palomino y la memoria histórica de los artistas en España”. En: Arte Barroco e ideal clásico. Aspectos del arte cortesano de la segunda mitad del siglo XVII. Ciclo de conferencias dirigido por Fernando Checa Cremades. Roma: SEACEX-Real Academia de España en Roma, pp. 89-111.

Bassegoda i Hugas, Bonaventura (2012): "Recensión al libro de Marta Cacho Casal, Francisco Pacheco y su libro de Retratos". En: "Biblioteca" Goya, 339, pp. 178-180.

Bassegoda i Hugas, Bonaventura (2015): "Libro de descripción de verdaderos retratos de ilustres y memorables varones". En: La fortuna de los libros: Madrid. Museo Lázaro Galdiano, cat. 4, pp. 37-41.

Cacho Casal, Marta (2010): "Portrait Drawings by Francisco Pacheco and the British Nineteenth-century Art Market”. En: Master Drawings, 4, 2010, pp. 447-455.

Cacho Casal, Marta (2011): Francisco Pacheco y su Libro de Retratos, Colección Premio Internacional Alfonso E. Pérez Sánchez. Madrid: Fundación Focus-Abengoa/Marcial Pons Historia.

Held, Jutta (1966): "Two portraits drawings by Goya". En: Master Drawings, vol. 4, nº 3, pp. 294-298.

Martínez Lara, Pedro (2012): Pablo de Céspedes. Estudio de los procesos de producción y asimilación entre Italia y España, entre el Renacimiento y el Barroco. Tesis doctoral inédita, II vols., Sevilla: Departamento de Historia del Arte: Universidad de Sevilla.

Muller, Priscilla E. (1996): "Pablo de Céspedes: a letter of 1577”. En: The Burlington Magazine, CXXXVIII, 1115 , pp. 89-91.

Muller, Priscilla E. (2006): Dibujos Españoles en la Hispanic Society of America. Del Siglo de Oro a Goya. Madrid: Museo Nacional del Prado.

\footnotetext{
${ }^{24}$ Sobre este tema véase el estudio comparativo que hace Cacho Casal, 2011: 217-223 acerca de la información que proporciona Pacheco en sus diferentes escritos y Palomino. Sobre la relación entre Pacheco y Céspedes es importante consultar también la tesis doctoral de Martínez Lara, 2012: I: 23-48.

${ }^{25}$ Bassegoda, 2000-2001: 208. Cacho Casal, 2010: 134-147 sin embargo piensa que el libro se desmembró y lo que hoy nos ha llegado es una parte del concebido por Pacheco por su desorden interno. Ha vuelto a cuestionar esta teoría Bassegoda, 2012: 178-80.

${ }^{26}$ Pacheco, 1990: 528.
}

Arch. esp. arte, LXXXIX, 353, ENERO-MARZO 2016, 77-84

ISSN: 0004-0428, eISSN: 1988-8511, doi: 10.3989/aearte.2016.06 
Pacheco, Francisco (1983): Libro de descripción de verdaderos Retratos de Ilustres y Memorables varones [1599]. Angulo Íñiguez, Diego (prólogo), Madrid: Previsión Española.

Pacheco, Francisco (1990): Arte de la Pintura. Bassegoda i Hugas, Bonaventura (ed.), Madrid: Cátedra.

Pérez Sánchez, Alfonso Emilio (1993): "Dos Goyas más y algunos "Goyas" menos”. En: Davis, Charles / Smith, Paul Julian (eds.): Art and Literature in Spain: 1600-1800. Studies in honour of Nigel Glendinning. Londres: Tamesis Book Limited, pp. 167-176.

Salas, Xavier de (1962): "Retratos de artistas españoles dibujados por Goya”. En: Goya, n 48, pp. 411-413.

Salas, Xavier de (1964): "Portraits of Spanish artists by Goya”. En: The Burlington Magazine, $\mathrm{n}^{\circ}$ 106, pp. 14-19.

Santarelli, Emilio (1870): Catalogo della Raccolta di Disegni Autografi antichi e moderni donata dal Prof. E. Santarelli alla R. Galleria di Firenze. Florencia: Cellini.

Santiago Páez, Elena (1966): Iconografía Hispana. Catálogo de los retratos de personajes españoles de la Biblioteca Nacional publicado por la sección de estampas. Madrid: Biblioteca Nacional.

Fecha de recepción: 21-VII-2015

Fecha de aceptación: 14-XII-2015

Arch. esp. arte, LXXXIX, 353, ENERO-MARZO 2016, 77-84 ISSN: 0004-0428, eISSN: 1988-8511, doi: 10.3989/aearte.2016.06 